\title{
Isozyme Variation in Silene pratensis: a Response to Different Environments
}

\author{
ONNO MASTENBROEK*, PAULINE HOGEWEG $\neq$, JAAP HERINGA $\neq$, GERARD J. NIEMANN $†$, \\ GERRIT VAN NIGTEVECHT* and JAN VAN BREDERODE* \\ * Department of Population and Evolutionary Biology, University of Utrecht, Padualaan 8, 3584 CH Utrecht, The Netherlands; \\ † Botanical Laboratory, University of Utrecht, Lange Nieuwstraat 106, 3572 PN Utrecht, The Netherlands; \\ ¥Bioinformatics Group, University of Utrecht, Padualaan 8, 3584 CH Utrecht, The Netherlands
}

Koy Word Index - Silene pratensis; Caryophyllaceae; isozyme variation; cluster analysis; flavone glycosides; flavone glycosylating genes; response to environment.

\begin{abstract}
The isozymes of nine enzyme systems were screened and the frequencies of the flavone glycosylating genes were determined in an outdoor experiment with 70 populations of European $S$. pratensis and an indoor experiment with 30 populations of the same species. Cluster analyses (using Ward's cluster criterion) were performed on all data sets. In the outdoor experiment, cluster analysis of the flavonoid data gave the same pattern that we obtained in an earlier survey of a larger set of populations and showed clearly that there are eight chemical races in European $S$. pratensis. No comparable geographic distribution could be found in the isozyme data set, although the dendrogram showed two very clearly separated groups. The two groups represented the two years in which the populations were grown. This result indicates that isozyme variation in European $S$. pratensis is largely determined by environmental factors. Observations on changes in isozyme patterns during ontogeny and on differences between indoor and outdoor grown plants of the same F2 crosses confirm this. Differences in isozyme patterns can be caused by very small differences in environment as is shown by the results of the indoor experiment, in which a slight gradient in environmental conditions was present in the greenhouses. The cluster analysis of the isozyme data from the indoor experiment revealed three distinct groups of populations that could be related to location within the greenhouses. As in the outdoor experiment, the dendrogram for the flavonoids gave the same geographic pattern as found with the earlier survey.
\end{abstract}

\section{Introduction}

Eight different chemical races have been distinguished in European Silene pratensis (Rafn) Godron and Gren. (Caryophyllaceae) using pattern recognition techniques on the geographic distribution of the alleles at three unlinked flavone glycosylating loci $[1,2]$. To determine whether these eight races are the result of selection or of other factors like drift, migration etc. we started to investigate the geographic distribution of other characters that are independent of the flavone glycosylating genes. Prentice [3] has shown that the variations in morphological characters of this species include a geographic component. Comparisons of the data sets on morphology and on flavone glycosylating genes revealed a striking similarity in the geographic distributions within both data sets [4]. A third data set for European $S$. pratensis was obtained by screening two sets of populations for isozymes as well as for flavonoids.

(Received 18 April 1983)
The results of this screening and the subsequent pattern analysis of the data are presented in this paper.

\section{Results}

Two separate experiments were carried out: one outdoors and one indoors. In the outdoor experiment 70 populations of $S$. pratensis were grown in two subsequent years (22 populations in 1980 and 48 in 1981 as well as six $S$. dioica populations, which were grown only in 1981). The indoor experiment was performed in 1982 with $30 \mathrm{~S}$. pratensis populations.

In the outdoor experiment a total of 83 isozyme bands were found for the nine enzyme systems investigated; the number of different bands and of the different patterns (i.e. phenotypes) are presented in Table 1. The investigation of the variation pattern was based on the frequencies of all bands observed in the different populations. No putative loci have been delimited, because there was no genetic analysis to support such delimitation. It can 
TABLE 1. THE 9 ENZYME SYSTEMS AND THEIR RESPECTIVE NUMBER OF DIFFERENT BANDS AND DIFFERENT PATTERNS

\begin{tabular}{llcl}
\hline Enzyme & EC-code & No. of bands & No. of patterns \\
\hline AcP & 3.1 .3 .2 & 9 & 34 \\
a-EST & 3.1 .1 & 12 & 68 \\
B-EST & 3.1 .1 & 10 & 62 \\
GOT & 2.6 .1 .1 & 6 & 10 \\
GPI & 5.3 .1 .9 & 6 & 14 \\
LAP & 3.4 .1 .1 & 5 & 10 \\
MDH & 1.1 .1 .37 & 8 & 21 \\
NADH DH & 1.6 .99 .3 & 13 & 63 \\
PO & 1.11 .1 .7 & 14 & 108 \\
\hline
\end{tabular}

be seen from Table 1 that peroxidase (PO) is the most variable enzyme, followed by NADH dehydrogenase (NADH DH) and both esterases. All the other enzyme systems show less variation. Two main clusters can be recognized in the dendrogram from the cluster analysis (see Fig. 1). The main division in the dendrogram is not correlated to the places of origin of the populations, nor did projection of the subclusters within the main clusters onto the map of Europe reveal a geographic pattern. Iterative character weighting [5] did not change the pattern. The optimum splitting level is at two clusters, with each of the clusters representing one of the years in which the experiment was performed. As these years had very different summers (1980: sunny, warm and dry and 1981: wet and cold [6]) it seems that the isozyme patterns are strongly influenced by environmental factors. From the KruskalWallis index we found that among the 10 most important isozyme bands, that determined the split in two clusters, were $6 \mathrm{PO}-$ and $3 \mathrm{NADH} \mathrm{DH}$ bands. This suggests that these enzyme systems are the most susceptible to external influences.

The dendrogram found for the flavone glycosylating gene frequencies of the populations of the outdoor experiment is presented in Fig. 2. The optimum splitting level is at five clusters. When these are projected on a map we find that the areas occupied by the five clusters coincide with the areas of the main clusters from our earlier analysis of the geographic distribution of the flavone glycosylating genes, in which we distinguished eight clusters using a larger set of populations (285) [2].

The major patterns in the flavonoid and isozyme data sets from the outdoor experiment appear to be unrelated. A geographic component is involved in the variation in flavone glycosylating genes, whereas this seems not to be the case with the isozyme variation. Some individual isozyme bands, however, may show geographic variation. The relationship between isozyme bands and the flavonoid cluster pattern was tested using the five flavonoid clusters for a supervised analysis of the isozyme data. None of the isozyme bands is distributed in these clusters in a statistically significant way (Kruskal-Wallis one way analysis of variance), nor can the flavonoid clusters be distinguished on a subset of the isozyme data (oligothetic characterisation of the clusters [7]).

The indoor experiment was performed with 30 populations, grown in two greenhouses. Each population was divided in two subpopulations for the analysis: females (subpopulation A) and males (B). The dendrogram for the flavone glycosylating gene frequencies again shows clusters which coincide both with the clusters of our earlier survey [2] and the clusters from the outdoor experiment presented in Fig. 2. Again the dendrograms for the isozyme band frequencies do not match the dendrogram for the flavone glycosylating gene frequencies. In the isozyme dendrograms the two sub-populations ( $A$ and $B$ ) of each population were more alike to each other than they were to any other (sub)population. This means that populations are very homogeneous and that the within-population variation for isozymes is much smaller than the between-population variation. Very often bands could be pointed out which only appeared in one or a few populations, using the Kruskal-Wallis fork analysis. The unweighted isozyme dendrogram (not illustrated) shows the presence of two main clusters. The first of these clusters presented the populations grown in twothirds of the two greenhouses used, while the second cluster consisted of the populations from the remaining part of the greenhouses. The first of these clusters was again split in two subclusters. The subclusters did not represent different parts of the greenhouses, but the places of origin of the populations involved: one subcluster presenting populations originating south of the Alps, the other the populations from north of these mountains. This geographic pattern, however, is very weak.

In order to investigate the clustering of populations of the back of the greenhouses against populations from the front we performed iterative character weighting [5] on the original 


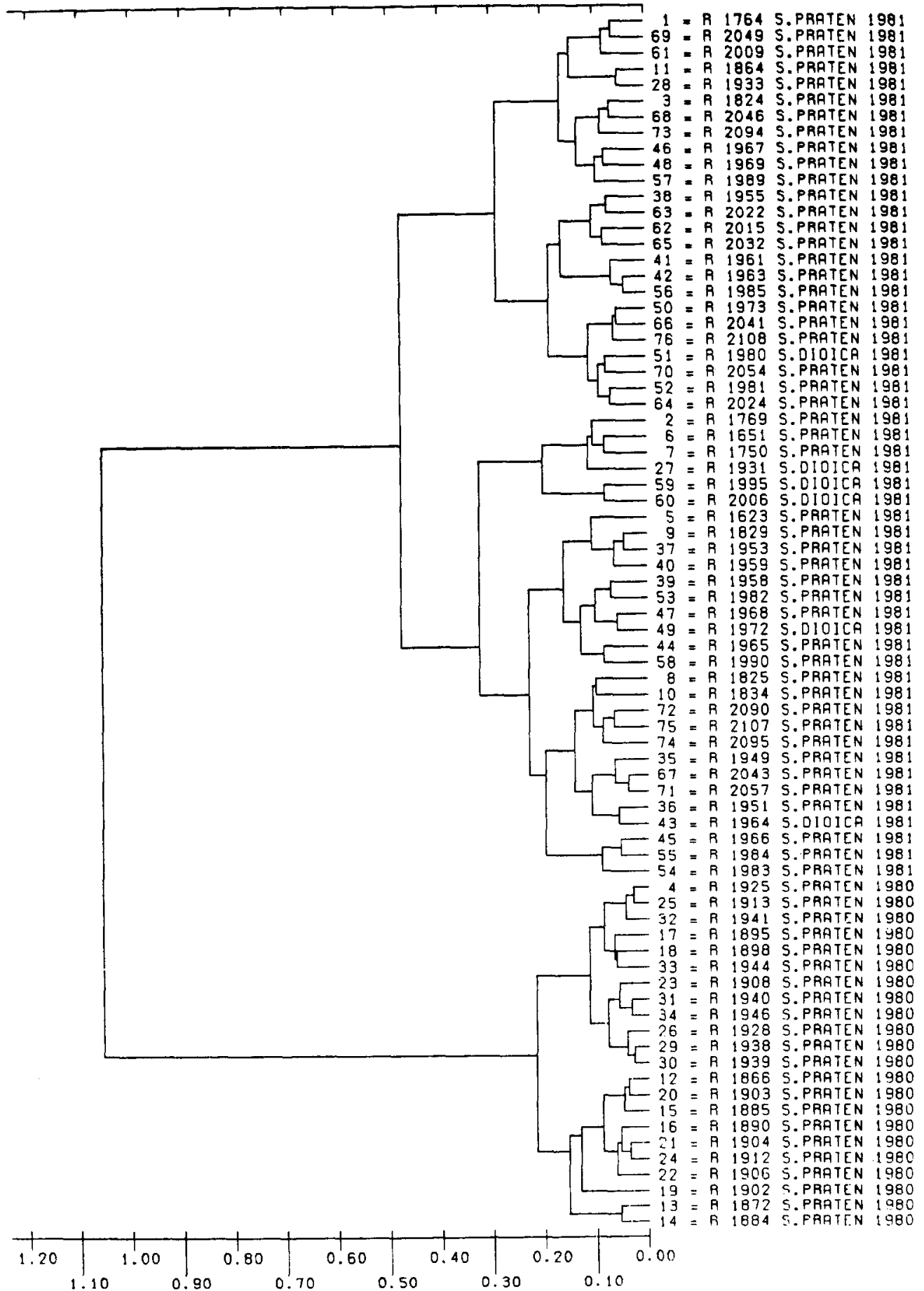

FIG. 1. THE DENDROGRAM OBTAINED BY CLUSTER ANALYSIS OF MEAN CHARACTER DIFFERENCES USING WARD'S CLUSTER CRITERION ON THE ISOZYMES OF THE POPULATIONS FROM THE OUTDOOR EXPERIMENT. As each population is labeled with the year in which it was grown, it will be clear that there is a separation between the two years in which the experiment was performed. Six $S$. dioica populations were included in this survey as well and they are indistinguishable from $S$. pratensis populations. 


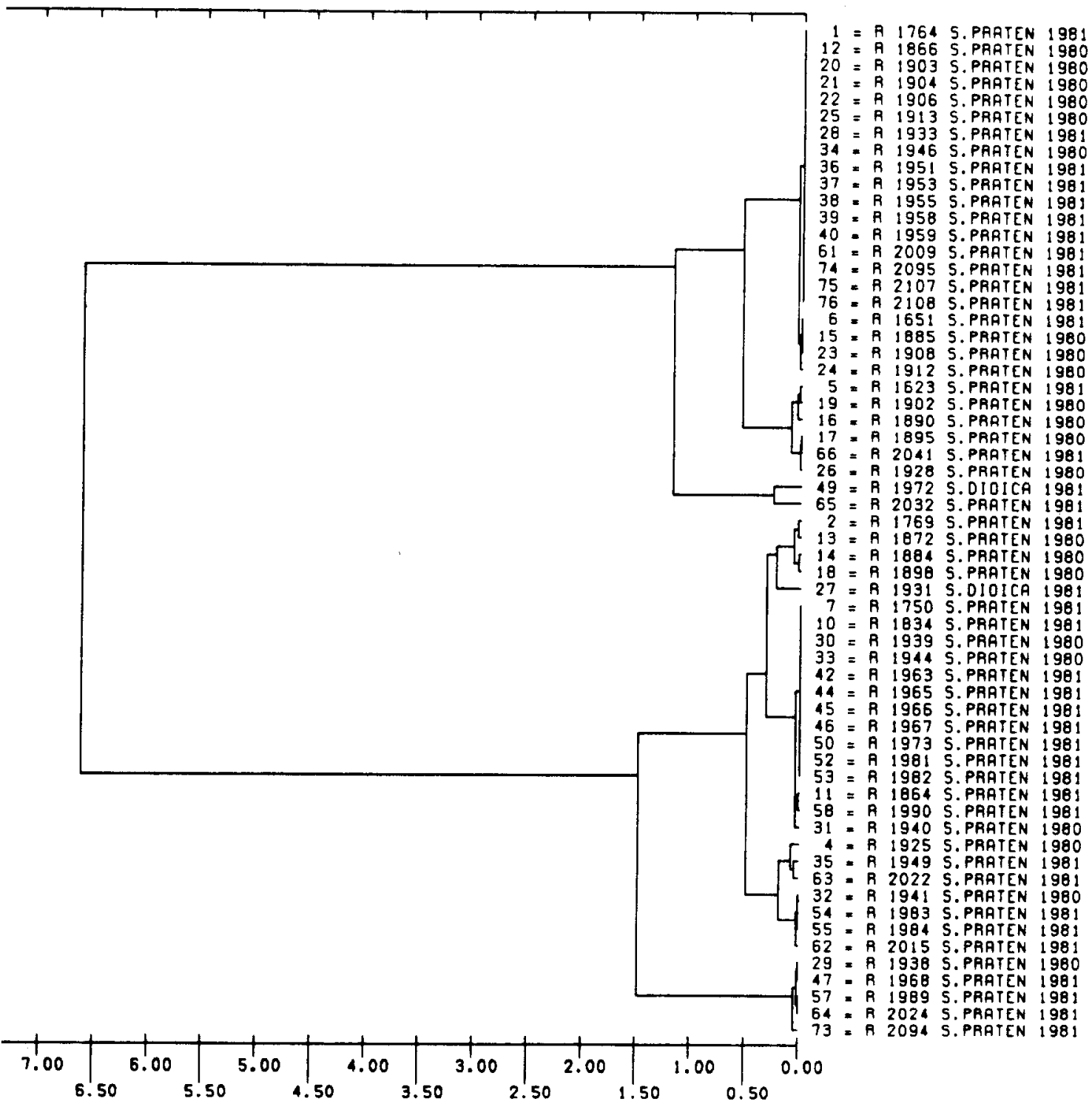

FIG. 2. THE DENDROGRAM OBTAINED ON THE FLAVONE GLYCOSYLATING GENES USING THE SAME TECHNIQUE AND THE SAME POPULATIONS AS IN FIG. 1. Only one $S$. dioica population could be included, because no gene-frequencies of the flavone-glycosylating genes were available for the other five populations. A comparison of Figs 1 and 2 show that the main clusters of the two dendrograms consist of very different populations. The year in which a population was grown does not influence the divisions in the dendrogram for the flavone-glycosylating genes. Another point that should be noted is that the various clusters in the second dendrogram are better separated than in the first dendrogram (see scales at the bottom of the figures). 


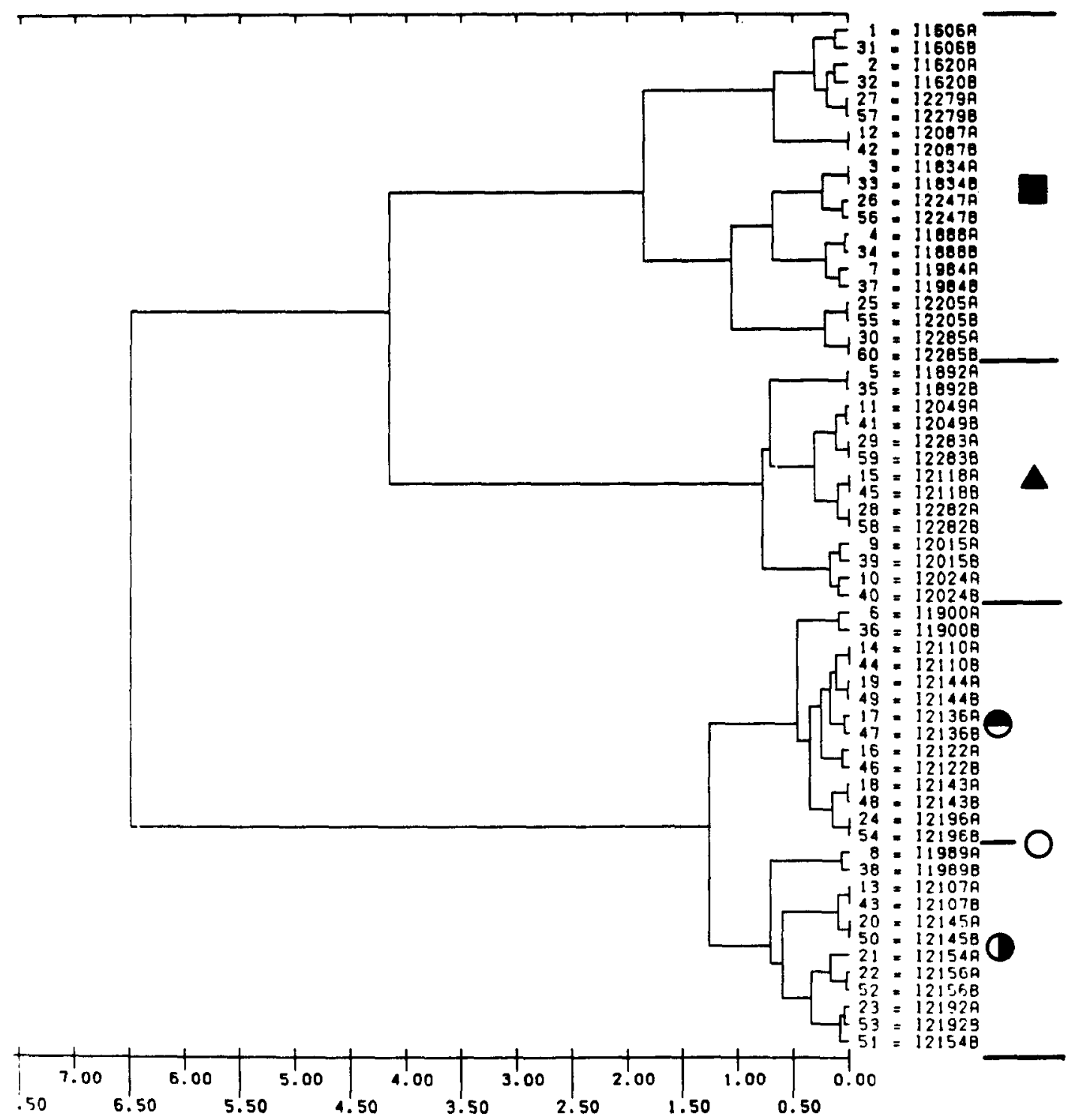

FIG. 3. THE DENDROGRAM FOR THE ISOZYME BAND FREQUENCIES OF THE POPULATIONS OF THE INDOOR EXPERIMENT. ThE POPUIAtions have been separated in ternale (A) and male (B) subpopulations, but these prove to be very homogenous as the differences between the subpopulations are always much smaller then between populations. The symbols representing the three main clusters and the two subclusters of the third cluster are used in Fig. 4. See text for further explanation.

dendrogram, generating the dendrogram in Fig. 3. Three main clusters could be recognized in this dendrogram, and again the two subpopulations of any population were more similar to each other than to other populations (with the exception of R2154A and B, Fig. 3). This time, however, each of the clusters represented populations from a different part from the greenhouses (see Fig. 4). The cluster consisting of the populations from the back remained the same as in the unweighted dendrogram. Of the two other clusters one represents the first part of the first greenhouse (no. 10 in Fig. 4) and the middle of the second greenhouse (no. 11), while the other mainly consists of 
a greenhouse 11 greenhouse 10

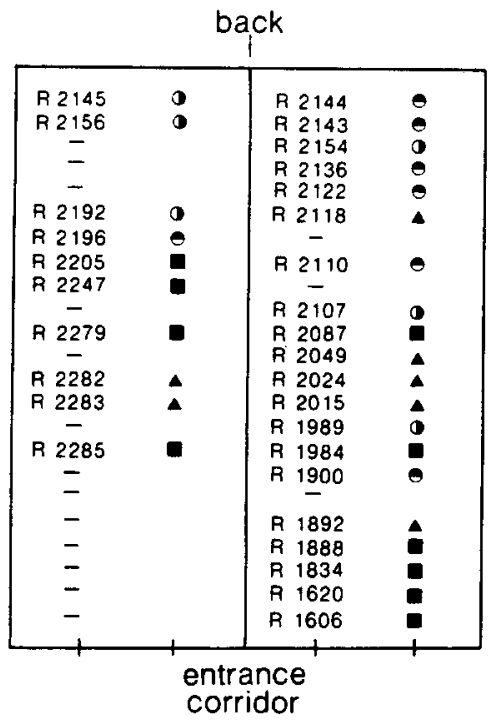

b

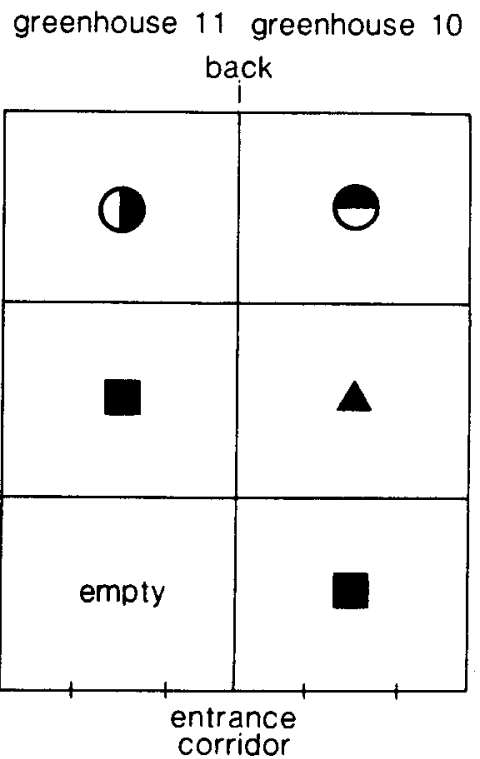

FIG. 4. A REPRESENTATION OF THE POSITION OF THE POPULATIONS IN THE TWO GREENHOUSES USED IN THE INDOOR EXPERIMENT. The symbol at each population represents the cluster that population belongs to (see fig. 3). It can be seen that the greenhouses can be subdivided in different parts, each represented by a (sub)cluster as is done in Fig. $4 b$.

populations of the middle of the first greenhouse. No trace is left of the geographic distribution. As the greenhouses show microvariation in climate (f.i. relative humidity and temperature), the conclusion must be that the isozyme patterns in $S$. pratensis are very sensitive to slight changes in the environment.

The isozyme bands which characterize best (Kruskal-Wallis one way analysis of variance) the main division of both the weighted and unweighted dendrogram, i.e. the split in the back part of the greenhouses versus the rest, are: PO-5, PO-8, ACP-2, $\alpha$-EST-1, $\alpha$-EST-2 and $\alpha$-EST-7. the same bands are involved in the second subdivision of the weighted dendrogram, which coincides with the frontal and middle parts of the greenhouse. Thus, these bands seem to respond very sensitively to environmental differences. In the unweighted dendrogram the second subdivision coincides with a geographic distribution, as mentioned above. Isozyme bands involved in this subdivision are apart from $\alpha$-EST-1, which is also involved in the first subdivision: PO-3, $\beta$-EST-10, NADH DH-8 and $\beta$-EST-1. The latter therefore seem to be less sensitive to environmental differences and reflect geographic distributions.

\section{Discussion}

It is clear from the dendrograms from both the outdoor and indoor experiments presented here and from their respective projections onto the map that there is no match in the distribution of isozyme variation and that in the flavone glycosylating genes. In contrast with the flavonoids and morphology $[2,4]$, isozyme variation shows no geographic distributions, but (at least the main part of it) shows a response to environmental factors. This is confirmed by two independent observations.

(a) Considerable variation in isozyme patterns has been found during the ontogeny of $S$. pratensis [8]. This variation is found both between and within the various developmental stages. Only within the stem leaf stage there was no variation; all leaves from a healthy mature plant having the same isozyme pattern. The isozyme variation during development did not show any regularity and it is therefore interpreted as being determined by the metabolic state of the individual rather than directly by the ontogenic phases [8]. A change in the metabolic state of the plant generates a quick response in the isozyme patterns, for instance different isozyme patterns in two subsequent 
rosette leaves. In our present study we used only stem leaves in order to minimize the variation due to differences during the developmental stages.

(b) We obtained an F2 generation by crossing plants that had different stem leaf isozyme patterns in our ontogeny experiment followed by crossings in the F1 generation. The objective of this was to determine the genetics of the isozyme systems investigated. Some of these F2's were grown during spring and summer in the greenhouse, while another batch was raised at the same time in the experimental plot. When we screened these plants for isozyme patterns, the plants that were grown outside had more and different bands than the plants inside. The environment inside the greenhouse is different from the one outside and this difference affects the metabolic state of the plants. An example of this is given by the formation of the flavonoid isoorientin from isovitexin, the basic flavonoid in S. pratensis. This formation is dependent on the light level [9] and plants grown inside mostly lack isoorientin due to a lower light level in the greenhouse. Again it seems likely that the plants differ in their metabolic states inside and outside the greenhouse and that this difference is responsible for the differences in isozyme patterns.

The response of the isozyme patterns to environmental fluctuations is rapid and the range of the short term variation is larger than differences in range between populations even between species. The latter is illustrated by the six $S$. dioica populations that were included in our survey (see Fig. 1). The $S$. dioica populations in our survey are indistinguishable from the $S$. pratensis populations in isozyme patterns. S. dioica is very closely related to $S$. pratensis and is characteristic of shaded rather than open conditions. When grown in an open plot $S$. dioica has the same isozyme patterns as $S$. pratensis lother differences, like flavonoid contents, of course, do not disappear).

The findings from our experiments lead to the following conclusions concerning the isozyme variation in European S. pratensis.

(a) The isozyme pattern of the part of the plant investigated is the result of the metabolic state of that part. The metabolic state is determined by the environment.

(b) Changes in the isozyme patterns are caused by changes in the enironment.

(c) Changes in the isozyme pattern can occur very quickly as the results of the ontogeny experiment show [8]. Two subsequent leaves can have very different patterns.

It is obvious from these points that populations must be grown in a controlled environment in order to be able to detect isozyme variation, that is a result of environmental influence. In an attempt to avoid such environmental influence our second experiment was set up in the greenhouse complex. From the dendrograms found for the isozyme variation (Fig. 3) it can be concluded that even under greenhouse conditions the environmental influence is still large. The populations growing at the back parts of the grenhouses are clearly separated from the other populations (Figs. 3 and 4). This fact could be explained by looking at the climate inside the greenhouses. The greenhouses used were not heated, while the adjoining corridor was heated day and night. In the period the plants developed to maturity (September) the temperatures dropped, especially at night. Therefore, there will have existed a temperature gradient of a few degrees centigrade in the greenhouse with its highest point near the adjoining heated corridor and its lowest point at the back of the greenhouse. This temperature gradient could have caused other gradients, e.g. humidity. This gradient will have been weak, but was steep enough for the populations at the back to react to it! One of the greenhouses (no. 11, Fig. 4) will have had a slightly higher mean day temperature as the sun can shine in all day, because it is not shaded by another greenhouse. After iterative character weighting we found we could divide the greenhouses in different parts, each respresented by different (sub)clusters (Figs. 3 and 4). So a fourth conclusion can be added to three already mentioned.

(d) The isozyme patterns in S. pratensis show a very marked reaction to even very slight changes in the environment.

In our survey of isozyme variation in European $S$. pratensis the pattern is largely determined by environmental factors. This does not mean that there is no genetic geographic variation at all, as is already indicated by the outcome of the unweighted dendrogram from the indoor experiment. To exclude any environmental influence it is necessary to grow the population under very rigidly controlled conditions.

The findings of a large environmental influence on isozyme variation indicates that one must be very careful in interpreting isozyme studies, 
performed for taxonomical, evolutionary or physiological purposes (see [10] for examples): such studies should always be accompanied by a sound genetic analysis. Before such an analysis can be performed on the isozymes of $S$. pratensis more must be known about the physiological background of the isozyme pattern changes. This physiological background could be elucidated by performing experiments with clones or related individuals like F1 and F2 progenies of selected crossings between plants with (under the same circumstances) different patterns for the various enzyme systems in various very rigidly controlled environments.

\section{Experimental}

Seed of 100 populations of $S$. pratensis and six populations of $S$. dioica was collected both by ourselves and by different systematic institutes in Europe. Care has been taken to establish that the seed was collected wild. A list of the populations with their places of origin and the frequencies of the isozyme bands may be obtained from 0 . Mastenbroek. The seeds from 70 populations were sown in our experimental plot in 2 successive years; the seed to 30 populations was sown in our greenhouse complex. As a rule 120 plants were grown for each population at the plot and 26 plants for the indoor populations. A stem leaf was collected from each mature plant [8]. The time needed to collect the leaves of all individuals was kept as short as possible, i.e. two to three days. The stem leaves were kept at $-20^{\circ}$ until they were used in electrophoresis. From each individual five to ten petals were gathered as well and stored in $70 \% \mathrm{MeOH}$ with $1 \% \mathrm{HCl}$ at $4^{\circ} \mathrm{C}$ to be able to determine the flavonoids.

Electrophoresis was performed as described by Mastenbroek et al. [8]. The populations were screened for nine different enzyme systems (see Table 1). Staining was according to Shaw and Prasad [11] except for MDH and NADH DH which were stained according to Mastenbroek et a. [8]. All the observed isozyme bands were numbered and the frequency of each band was determined in each population.

The flavonoid content of each individual was determined using paper chromatography as described by Mastenbroek et al. [1]. For each population the frequency of the flavone glycosylating genes was estimated using the Hardy-Weinberg equation for genetic equilibrium.

The frequency for each isozyme band per population was determined by dividing the number of individuals in the population possessing that band by the total number of plants of the population. These frequencies were subjected to agglomerative cluster analysis (based on mean character differences [12] of isozyme band trequencies and using Ward's cluster criterion [13]). Optimal splitting levels were determined for the dendrogram [5] and the contribution of the various isozyme band frequencies to the separation of clusters were determined using the Kruskal-Wallis index. The same technique were used independently on the flavone glycosylating gene frequencies of the populations. Other techniques that were used on the isozyme band frequencies were iterative character weighting [5] and oliogothetic characterisation of clusters [7]. The pattern analysis was done using BIOPAT [14].

The weather reports [6] were sent to us regularly by the Royal Dutch Institute for Meteorology (KNMI) situated in De Bilt, less than $2 \mathrm{~km}$ from our experimental plot.

Acknowledgements - We wish to thank Ir. $H$. van Koppen for his help in transferring the data between computers and Dr. H. C. Prentice for her comments on the English.

\section{References}

1. Mastenbroek, O., Maas, J. W., Brederode, J. van, Niemann, G. J. and Nigtevecht, G. van (1982) Genetica 59, 139.

2. Mastenbroek, O., Hogeweg, P., Brederode, J. van and Nigtevecht, G. van (1983) Biochem. Syst. Ecol. 11, 91.

3. Prentice, H. C. (1979) Bot. J. Linn. Soc. 78, 181.

4. Mastenbroek, O., Prentice, H. C., Kamps-Heinsbroek, R., Brederode, J. van, Niemann, G. J. and Nigtevecht, G. van (1983) Plant Syst. Evol. 141, 257.

5. Hogeweg, P. (1976) Comput. Biol. Med. 6, 199.

6. KNMI weather reports, KNMI. De Bilt.

7. Hogeweg, P. and Hesper, B. (1981) Pattern Recognition 14, 131 .

8. Mastenbroek, O., Brederode, J. van, Niemann, G. J. and Nigtevecht, G. van (1981) Biochem. Physiol, Pflanz. 176,584

9. Brederode, J. van, Kamps-Heinsbroek, R. and Mastenbroek, 0. (1982) Z. Pflanzenphysiol. 106, 43.

10. Gottlieb, L. D. (1981) Prog. Phytochem. 7, 1.

11. Shaw, R. C. and Prasad, R. (1970) Biochem. Genet. 4, 297.

12. Bock, H. H. (1974) Automatische Klassifikation. van den Hoeck und Ruprecht, Gottingen.

13. Ward, J. H. (1963) J. Am. Stat. Assoc. 58, 236.

14. Hogeweg, P. and Hesper, B. (1972) BIOPAT, Program system for biological pattern analysis."Bioinformatica, Utrecht. 